\title{
On the nature of clumps in debris disks ${ }^{\star}$
}

\author{
A. V. Krivov ${ }^{1}$, M. Queck ${ }^{1}$, T. Löhne ${ }^{1}$, and M. Sremčević \\ 1 Astrophysikalisches Institut, Friedrich-Schiller-Universität Jena, Schillergäßchen 2-3, 07745 Jena, Germany \\ e-mail: krivov@astro.uni-jena.de \\ 2 LASP, University of Colorado, 1234 Innovation Drive, Boulder, CO 80303, USA
}

Received 10 May 2006 / Accepted 20 September 2006

\begin{abstract}
The azimuthal substructure observed in some debris disks, as exemplified by $\epsilon$ Eridani, is usually attributed to resonances with embedded planets. In a standard scenario, the Poynting-Robertson force, possibly enhanced by the stellar wind drag, is responsible for the delivery of dust from outer regions of the disk to locations of external mean-motion planetary resonances; the captured particles then create characteristic "clumps". Alternatively, it has been suggested that the observed features in systems like $\epsilon$ Eri may stem from populations of planetesimals that have been captured in resonances with the planet, such as Plutinos and Trojans in the solar system. A large fraction of dust produced by these bodies would stay locked in the same resonance, creating the dusty clumps. To investigate both scenarios and their applicability limits for a wide range of stars, planets, disk densities, and planetesimal families we construct simple analytic models for both scenarios. In particular, we show that the first scenario works for disks with the pole-on optical depths below about $\sim 10^{-4}-10^{-5}$. Above this optical depth level, the first scenario will generate a narrow resonant ring with a hardly visible azimuthal structure, rather than clumps. It is slightly more efficient for more luminous/massive stars, more massive planets, and planets with smaller orbital radii, but all these dependencies are weak. The efficiency of the second scenario is proportional to the mass of the resonant planetesimal family, as example, a family with a total mass of $\sim 0.01$ to 0.1 Earth masses could be sufficient to account for the clumps of $\epsilon$ Eridani. The brightness of the clumps produced by the second scenario increases with the decreasing luminosity of the star, increasing planetary mass, and decreasing orbital radius of the planet. All these dependencies are much stronger than in the first scenario. Models of the second scenario are quantitatively more uncertain than those of the first one, because they are very sensitive to poorly known properties of the collisional grinding process.
\end{abstract}

Key words. planetary systems: formation - circumstellar matter - meteors, meteoroids - celestial mechanics - stars: individual: $\epsilon$ Eri.

\section{Statement of the problem}

Various kinds of structures - offsets, wing asymmetries, warps, voids, clumps, rings, spirals, inner gaps - are either seen in the images of resolved debris disks or have been retrieved from analyses of spectral energy distributions. Accordingly, a variety of mechanisms have been suggested to explain the structure formation (see, e.g. Augereau 2004, for a recent review). Embedded planets can create structures through secular (long-term) perturbations (Mouillet et al. 1997), by resonant perturbations (Liou \& Zook 1999), as well as by gravitational scattering of the disk particles (Moro-Martín \& Malhotra 2005). Some features may stem from recent stellar flybys (Kalas et al. 2001); others may reflect recent major collisional events between large planetesimals (Wyatt \& Dent 2002; Kenyon \& Bromley 2005; Grigorieva et al. 2007)

Here we address the azimuthal substructure seen in several disks, best exemplified by the 0.85 Gyr-old K2V star, $\epsilon$ Eridani. Submillimeter images obtained with JCMT/SCUBA sighted a few bright blobs or clumps in this nearly pole-on disk (Greaves et al. 1998). New observations of the same disk (Greaves et al. 2005) revealed signs of the disk rotation, demonstrating that three out of six clumps are real and indicating that the observed rotation rate is compatible with the Keplerian velocity at the location of the clumps. A similar clumpy structure has recently been found in the pole-on disk around another $1 \mathrm{Gyr}$-old

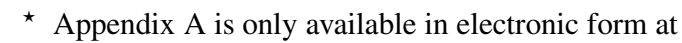
http: //www . aanda.org
K star, HD 53143 (Kalas et al. 2006). Clumps have also been reported for other disks seen at moderate inclinations, most notably Fomalhaut (Holland et al. 2003) and Vega (Holland et al. 1998; Wilner et al. 2002).

It is natural to attribute the formation of blobs to the resonant perturbations exerted on dust grains by a planet. But how can the resonances create the clumps? One mechanism, originally proposed by Gold (1975) and discussed very intensively in the past decade, which we call scenario I, can be summarized as follows. Dust particles in dilute debris disks are known to steadily migrate inwards due to the Poynting-Robertson (P-R) force, possibly enhanced by the stellar wind (e.g. Weidenschilling \& Jackson 1993; Beaugé \& Ferraz-Mello 1994; Liou \& Zook 1997). If there is a planet in the disk, the particles reach locations of exterior mean-motion resonances (MMRs) with the planet and get trapped, yielding characteristic density patterns.

Liou et al. (1996) and Liou \& Zook (1999) applied this idea to the solar system. By modeling perturbations induced by jovian planets on the Edgeworth-Kuiper Belt dust disk, they found efficient resonant trapping of dust by Neptune (which produces arcs of dust co-orbital with the planet) and efficient ejection of dust out of the solar system by Jupiter and Saturn. Were the solar system observed from outside, the presence of at least Neptune and Jupiter would be obvious merely from analyzing images of the dust disk. While the large-scale clumps on the outskirts of the solar system still escape observational detection, there is one, so far the only, case where scenario I is observed at work: the asymmetric resonant ring of asteroidal dust around the Earth 
orbit. Predicted by Jackson \& Zook (1989), it was identified in IRAS (Dermott et al. 1994) and COBE/DIRBE data (Reach et al. 1995). Search of a similar ring around the Mars orbit was not successful, however (Kuchner et al. 2000).

In the case of $\epsilon$ Eri, Liou et al. (2000), Ozernoy et al. (2000), Quillen \& Thorndike (2002), and Deller \& Maddison (2005) have tried to find particular orbital parameters and masses of a planet, or planets, that may reproduce the observed substructure in the disk. A systematic overview of resonant structures that a single planet can form in a debris disk through this mechanism was painted by Kuchner \& Holman (2003).

A major problem with this mechanism is that catastrophic grain-grain collisions may smear out the planet-induced structure already at moderate optical depths, because the collisional lifetimes appear to be shorter than the P-R timescale that determines the dust injection rate into the resonance (Lecavelier des Etangs et al. 1996; Lagrange et al. 2000). Wyatt (2003, 2006) suggested an alternative scenario, hereinafter scenario II, in which the clumps stem from planetesimals that have been captured in planetary resonances, possibly as a result of the planet's migration. Such resonant populations have long been known in the solar system - Plutinos are locked in the 3:2 MMR with Neptune, while Greeks and Trojans reside in the primary resonance with Jupiter. A large fraction of the dust which these bodies produce may stay locked in the same resonance and could well account for the observed clumps.

In this paper, we make a comparative analysis of both scenarios and estimate typical timescales and efficiency of both scenarios with simple analytic models. The goal is to investigate and compare the efficiency of both scenarios for a wide range of essential parameters, such as the mass of the central star, mass and orbital radius of the embedded planet, as well as the optical depth of the "background" disk (in the first scenario) and properties of the resonant family of planetesimals (in the second one). Scenarios I and II are considered in Sects. 2 and 3, respectively. Section 4 contains our conclusions and a discussion.

\section{Scenario I}

We start with an analysis of scenario I, in which dust particles are brought from afar to resonance locations by the P-R effect and are captured in resonances. A resonance implies a $(p+q)$ : $p$-commensurability of the mean motions of the planet and the grain. If $a_{\mathrm{p}}$ is the semimajor axis of the planet orbit, the resonant value of the grain's orbital semimajor axis is

$a=a_{\mathrm{p}}(1-\beta)^{1 / 3}\left(\frac{p+q}{p}\right)^{2 / 3}$,

with $\beta$ being the radiation pressure to gravity ratio for the dust grain (assumed to be a perfect absorber):

$\beta=0.57\left(\frac{L_{*}}{L_{\odot}}\right)\left(\frac{M_{\odot}}{M_{*}}\right)\left(\frac{1 \mathrm{~g} \mathrm{~cm}^{-3}}{\rho}\right)\left(\frac{1 \mu \mathrm{m}}{s}\right)$,

where $L_{*} / L_{\odot}$ and $M_{*} / M_{\odot}$ are luminosity and mass of the star in solar units and $s$ is the grain radius. In what follows, we treat $M_{*}$ as a parameter and calculate luminosity by using the standard $L_{*} \propto M_{*}^{4}$ relation for main-sequence stars. Throughout the paper, the bulk density of $2 \mathrm{~g} \mathrm{~cm}^{-3}$ is adopted. To keep our treatment simple, we confine our analysis to a circular planetary orbit. The typical resonant dynamics can then be summarized as follows (e.g. Weidenschilling \& Jackson 1993; Beaugé \& Ferraz-Mello 1994; Liou \& Zook 1997). When trapped, a dust grain preserves the semimajor axis. If $\lambda$ and $\lambda_{\mathrm{p}}$ are longitudes of the grain and planet, respectively, and $\tilde{\omega}$ is the longitude of pericenter of the grain orbit, the resonant argument

$\Phi=(p+q) \lambda-p \lambda_{\mathrm{p}}-q \tilde{\omega}$

librates with an amplitude $A$ around the value $\approx 180^{\circ}$ (cf. Kuchner \& Holman 2003; Wyatt 2003, 2006). The eccentricity gradually pumps up. For initially circular orbits (Liou \& Zook 1997)

$e^{2}(t)=\frac{q}{3 p}\left[1-\exp \left(-\frac{3 p}{p+q} B t\right)\right]$,

where

$B=\frac{2 G M_{*} \beta}{c a^{2}}$

$G M_{*}$ standing for the gravitational parameter of the central star and $c$ for the speed of light. When the eccentricity reaches a certain value $e_{\text {res }}$, the particle becomes a planet-crosser and shortly thereafter either collides with the planet or gets ejected out of the resonance zone after a close encounter. The orbital inclination $i$ exponentially decreases on the same timescale as $e$ increases; here we assume $0 \leq i \leq \epsilon$, where $\epsilon$ is a (small) semi-opening angle of the disk.

Note that some grains that used to be trapped in a resonance may then continue migrating toward the star, instead of being ejected from the system. This is, however, more typical of resonances with very low-mass planets - e.g. in the Earth resonant ring mentioned above. For such planets, overlapping of first-order resonances and the onset of chaos, as well as external perturbations by other planets, may also play a role, making the dynamics more complicated (Marzari \& Vanzani 1994). Since dust structures induced by terrestrial planets around other stars are far beyond observational limits, our analysis is confined to at least Neptune/Uranus-mass planets and thus ignores any temporary resonant capture of particles.

To construct a simple model, we choose a "typical" grain radius $s$ and a corresponding $\beta$-value. This is the size of the grains that compromises the cross section-dominating size and the size for which the probability of capture in the resonance $\left(p_{\text {res }}\right.$ below) becomes significant. The former corresponds to $\beta$ of several tenths (Krivov et al. 2000; Thébault et al. 2003; Krivov et al. 2006). The latter requires $\beta \lesssim 0.1$ (e.g. Liou et al. 1996; Liou $\&$ Zook 1999); the smaller $\beta$, the larger $p_{\text {res. }}$ In our model, we treat $\beta$ as a free parameter and use $\beta=0.1$ as a default value in numerical examples.

For a certain MMR and a given $\beta$ ratio, the resonant value of semimajor axis $a$ is given by Eq. (1). The particles drift towards the star by P-R drag. Assume that $\dot{n}^{+}$grains cross $r=a$ per unit time. Let $p_{\text {res }}$ be the probability of trapping in the resonance. Its value depends on size, initial eccentricity, and the inclination of the grain orbit, planet's mass $M_{\mathrm{p}}$, and varies from one resonance to another (e.g. Weidenschilling \& Jackson 1993; Beaugé \& Ferraz-Mello 1994; Liou et al. 1996; Liou \& Zook 1997). For instance, Liou \& Zook (1999) report that $p_{\text {res }}$ increases from about 0.2 for $\beta=0.4$ to about 0.5 for $\beta=0.05$ for the Edgeworth-Kuiper belt particles' trapping by Neptune. Inside a certain range of these parameters, however, $p_{\text {res }}$ is not very sensitive to their variation. We performed a number of numerical integrations, which we do not describe here, because the technique is very well known (e.g. Weidenschilling \& Jackson 1993; Beaugé \& Ferraz-Mello 1994; Liou \& Zook 1997; Quillen \& Thorndike 2002; Deller \& Maddison 2005). For the parameters 


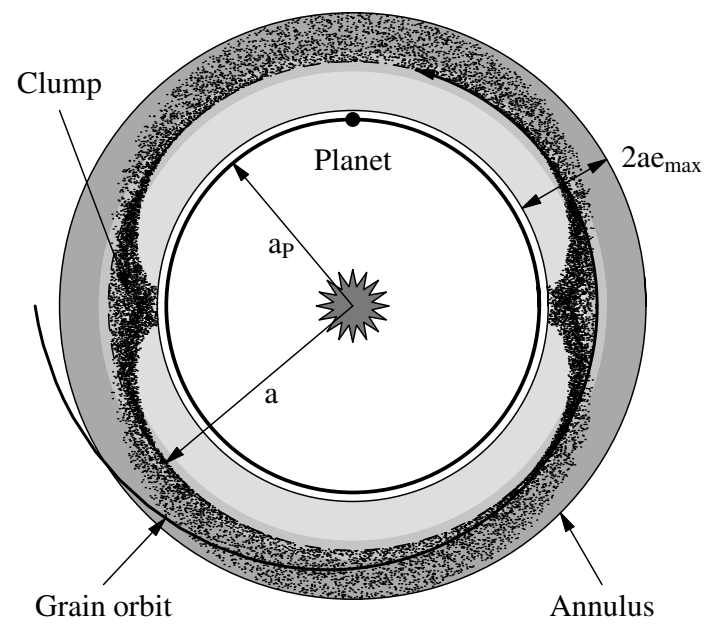

Fig. 1. Schematic of the resonant and non-resonant components of the dust disk in the vicinity of a given resonance. The particular resonant population with two clumps shown here corresponds to the 3:2-MMR with $e_{\max }=0.2$ and $A=20^{\circ}$.

of the $\epsilon$ Eri system, with initial eccentricities $\lesssim 0.1$, inclinations below $10^{\circ}$, and a planet at $40 \mathrm{AU}$ with 0.1 Jupiter mass, the trapping probability is between $10 \%$ and almost $100 \%$ for many resonances $\left(2: 1,3: 2,8: 5,5: 3,9: 5\right.$, and others). In our model, $p_{\text {res }}$ is a parameter. In numerical examples, $p_{\text {res }}=0.5$ is used as a default value.

Besides $p_{\text {res}}$, another quantity we need is $e_{\text {res }}$, the value of orbital eccentricity that the captured grains have to develop to quit the resonance. On the basis of the papers cited above and our own numerical integrations, the typical values lie between 0.1 and 0.4. Like $\beta$ and $p_{\text {res }}, e_{\text {res }}$ is a model parameter. As a "standard" value, we adopt $e_{\text {res }}=0.2$. Note that grains do not necessarily reach $e=e_{\text {res }}$. Let us denote by $T_{\text {res }}$ the time interval in which $e_{\text {res }}$ is attained. As the particles are lost not only because they pump up the orbital eccentricities to the resonancequitting value $e_{\text {res }}$, but also are eliminated by mutual collisions, their mean actual lifetime $T$ is shorter than $T_{\text {res }}$. Therefore, their actual maximum eccentricity $e_{\max }$, i.e. the maximum eccentricity achieved by the particles in their lifetime $T$, will be smaller than $e_{\text {res. }}$.

We now introduce an annulus in the disk around the location of a given MMR (Fig. 1), defined as follows. We require that the annulus width matches the maximum radial excursion of the trapped particles: from $a\left(1+e_{\max }\right)$ to $a\left(1-e_{\max }\right)$. At any instant in time, the annulus contains two populations of particles. One is a non-resonant "background" population of particles, including those that have not reached the resonance location yet, as well as those that drifted through it without getting trapped. It might also include grains that continue drifting toward the star after quitting resonance. If such grains are present in sufficient amounts, $p_{\text {res }}$ should be re-defined to account for them. All these particles have a uniform azimuthal distribution. Another population is an unevenly distributed (clumpy) population of resonant grains. Hereinafter, all quantities with subscript " 0 " characterize the background population and those without subscript the resonant population. For instance, $n_{0}$ and $n$ stand for the total number of background grains and resonant grains in the annulus, respectively.

We now construct a simple kinetic model. By applying any standard method of the kinetic theory, or simply by "counting" the particles injected into and destroyed in the annulus per unit time, one finds the balance equation:

$$
\frac{\mathrm{d} n}{\mathrm{~d} t}=\dot{n}^{+} p_{\text {res }}-\frac{n}{T_{\text {res }}}-\frac{n}{T_{\text {coll }}^{0}}-\frac{n}{T_{\text {coll }}},
$$

where $T$ s are timescales as explained below. As $n$ does not have a grain size/mass as argument, Eq. (6) can be referred to as the Boltzmann equation. Since $n$ does not have any grain velocity argument either, it can also be termed the Smoluchowski equation. See, e.g., Krivov et al. (2005) and references therein for a detailed discussion of a general form of the balance equation.

The resonant population implies several natural timescales associated with the terms on the right-hand side of Eq. (6). The first is $T_{\mathrm{PR}}$, the P-R time to drift from $a\left(1+e_{\max }\right)$ to $a\left(1-e_{\max }\right)$. The second is $T_{\text {res }}$, the time of eccentricity pumping to the value $e_{\text {res }}$ for trapped grains. It can also be treated as the maximum time of residence in the resonance for trapped grains. Finally, $T_{\text {coll }}^{0}$ and $T_{\text {coll }}$ are the lifetimes of the resonant grains against collisions with background grains and other resonant grains, respectively.

The actual lifetime of grains as determined by all sinks together is given by

$$
\frac{1}{T} \equiv \frac{1}{T_{\text {res }}}+\frac{1}{T_{\text {coll }}^{0}}+\frac{1}{T_{\text {coll }}}
$$

and the maximum eccentricity achieved by the particles in the time $T$, as follows from Eq. (4) for small $e_{\max }$, is

$e_{\max } \equiv e(T) \approx \sqrt{\frac{q}{p+q} B T}$.

We return to Eq. (6) and begin with its first term. The steadystate number of non-resonant grains in the annulus, $n_{0}$, is

$n_{0}=\dot{n}^{+}\left(1-\frac{p_{\text {res }}}{2}\right) T_{\mathrm{PR}}$

where the P-R drift time is given by (e.g. Burns et al. 1979)

$T_{\mathrm{PR}}=\frac{4 e_{\max }}{B}$.

Equation (9) reflects the fact that the outer half of the annulus, the region between $a\left(1+e_{\max }\right)$ and $a$, is always filled by the background grains, regardless of whether some of them get captured in the resonance upon arrival at $a$. In contrast, the number of grains in the inner half, between $a$ and $a\left(1-e_{\max }\right)$, lies between zero (if $p_{\text {res }}=1$ ) and the number of grains in the outer half (if $p_{\text {res }}=0$ ). These two regions are shown in Fig. 1 with dark and light grey, respectively. We note, however, that this division of the annulus into the outer and inner parts is done in terms of the semimajor axis and not in real space. Since grain orbits have non-zero eccentricities, no sharp boundary between the two halves exists in terms of distance, i.e. at $r=a$. The boundaries of the whole annulus will also be somewhat fuzzy.

We introduce the normal optical depth $\tau_{0}$ of the non-resonant population in the outer part of the annulus, which is not affected by the resonance. It is a product of the number of grains in the outer part and the cross section of a grain, divided by the area of the outer half of the annulus:

$\tau_{0}=\frac{\dot{n}^{+} T_{\mathrm{PR}} / 2 \cdot \pi s^{2}}{2 \pi a^{2} e_{\max }}$.

Substituting Eq. (10) and solving for $\dot{n}^{+}$results in

$\dot{n}^{+}=B\left(\frac{a}{s}\right)^{2} \tau_{0}$. 
Thus $\dot{n}^{+}$and thereby the first term in Eq. (6) are expressed through an "observable" quantity, the background optical depth $\tau_{0}$.

The resonant pumping time $T_{\text {res }}$ in the second term of Eq. (6) comes from Eq. (4). For small $e_{\text {res }}$,

$T_{\mathrm{res}} \approx \frac{p+q}{q} \frac{e_{\mathrm{res}}^{2}}{B}$.

Finally, the collisional lifetimes $T_{\text {coll }}^{0}$ and $T_{\text {coll }}$ in Eq. (6) should be estimated. General expressions are

$T_{\text {coll }}^{0}=\frac{V_{0}}{n_{0} \sigma v_{0}} \quad$ and $\quad T_{\text {coll }}=\frac{V}{n \sigma v}$,

where $\sigma=4 \pi s^{2}$ is the collision cross section of the like-sized particles, $v_{0}$ the mean impact velocity between background and resonant grains, $V_{0}$ the effective volume of their interaction specified below, and $v$ and $V$ are similar quantities for mutual collisions of resonant grains.

Consider $T_{\text {coll }}^{0}$ and the third term of Eq. (6) first. The interaction volume is given by (see, e.g., Appendix in Krivov et al. 2006)

$V_{0}=(2 \pi a)^{2} h|\sin \gamma|$,

where $h$ is the disk thickness ( $h=2 a \sin \epsilon$ for a disk with the semi-opening angle $\epsilon$ ) and $\gamma$ the angle between the orbits of the particles locked in the resonance and the orbits of non-resonant grains at their intersection point. With

$v_{\mathrm{k}}=\left(G M_{*}(1-\beta) / a\right)^{1 / 2}$

being the Keplerian circular velocity and neglecting corrections due to small eccentricities and inclinations, we have

$v_{0} \approx v_{\mathrm{k}}|\sin \gamma|$.

Thus the ratio $V_{0} / v_{0}$ in the first Eq. (14) is independent of eccentricities and inclinations, as long as they are small, and that equation simplifies to

$T_{\text {coll }}^{0}=\frac{1}{n_{0}} \frac{(2 \pi a)^{2} h}{\sigma v_{\mathrm{k}}} \equiv \frac{1}{n_{0}} \hat{T}_{\mathrm{coll}}$.

We also note that, in the monosize model considered, $T_{\text {coll }}^{0}$ is nearly independent of the particle size chosen. This is seen from Eqs. (9) and (11), if we neglect a weak dependence of $v_{\mathrm{k}}$ on the grain radius.

To compute $T_{\text {coll }}$ for the fourth term of Eq. (6), we need the interaction volume $V$ and the average impact velocity $v$ for mutual collisions between the particles in the resonant clumps. Accurate calculations for this case are not easy, because the resonant population has a distribution of eccentricities from 0 to $e_{\max }$ and a highly non-uniform distribution of apsidal lines. There is no guarantee therefore that the particle-in-a-box-like estimates we employed for $T_{\text {coll }}^{0}$ would yield correct results. A full solution of this problem, which may have a number of applications to resonant systems, is beyond the scope of this paper. Our calculations (Queck et al., in prep.) show, however, that an analog of Eq. (18),

$T_{\text {coll }}=\frac{1}{n} \frac{(2 \pi a)^{2} h}{\sigma v_{\mathrm{k}}}=\frac{1}{n} \hat{T}_{\text {coll }}$,

provides results that are within a factor of two from the "true" values.

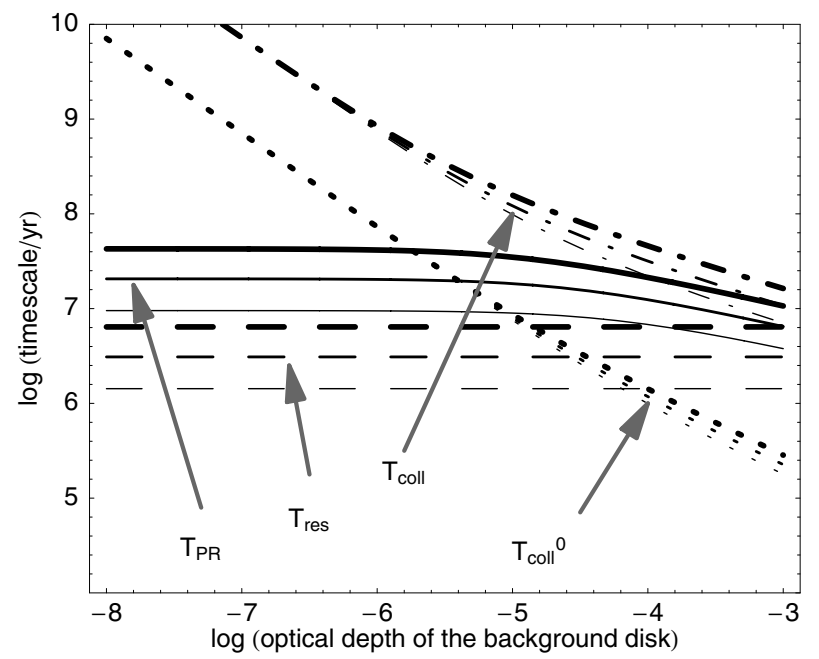

Fig. 2. Typical timescales as functions of the normal optical depth $\tau_{0}$ of the non-resonant disk: $T_{\mathrm{PR}}$ (solid), $T_{\text {res }}$ (dashed), $T_{\text {coll }}^{0}$ (dotted), $T_{\text {coll }}$ (dash-dotted). Thick, medium, and thin lines: $\beta=0.05,0.10$, and 0.20 , respectively. These values correspond to $s=2.9,1.5$, and $0.7 \mu \mathrm{m}$. The collisional time $T_{\text {coll }}^{0}$ is nearly the same for all three values (see text).

Thus all characteristic timescales, and all four terms on the right-hand side of Eq. (6), are now determined. A little technical complication arises from the fact that $T_{\text {coll }}^{0}$ in Eq. (18) depends through $n_{0}$ on $e_{\max }$ (Eqs. (9) and (10)) which, in turn, depends on $T$ and thus back on $T_{\text {coll }}^{0}$ (Eqs. (7) and (8)). Actual calculations have therefore been done iteratively, with $e_{\text {res }}$ as the first approximation for $e_{\max }$.

For illustrative purposes, we choose the parameters suitable for the $\epsilon$ Eridani disk: $M_{*}=0.8 M_{\odot}$, the presumed planet at $a_{\mathrm{p}}=40 \mathrm{AU}$ (Liou et al. 2000; Ozernoy et al. 2000; Quillen \& Thorndike 2002; Deller \& Maddison 2005). The collisional lifetime (18) is proportional to the semi-opening angle of the disk $\epsilon$, which is unconstrained. By analogy with other debris disks seen edge-on ( $\beta$ Pic, AU Mic), we take $\epsilon=0.1=6^{\circ}$. We choose 3:2-MMR, but the results are very similar for other low-degree, low-order resonances. Figure 2 plots the timescales as functions of the optical depth $\tau_{0}$ of the background disk, showing that the collisional lifetime $T_{\text {coll }}^{0}$ is shorter than the other timescales for disks with roughly $\tau_{0} \gtrsim 10^{-5}$, which is the case for $\epsilon$ Eri.

Figure 3 shows how the maximum orbital eccentricity $e_{\max }$ of resonant particles decreases with increasing optical depth. It also shows that, for denser disks, $e_{\max }$ depends only weakly on the maximum value $e_{\text {res }}$ determined by the resonant dynamics. For $\tau_{0} \sim 10^{-4}$, the resulting value $e_{\max }$ is close to 0.1 , regardless of $e_{\text {res }}$. For such low eccentricities, the resonant population will look like a narrow ring without azimuthal structure, not like clumps. The dustier the disk, the narrower the resulting ring (for planets in near-circular orbits), and the less pronounced the azimuthal structure. Therefore, prior to any calculation of the clumps' optical depth, we can conclude that in sufficiently dusty disks, scenario I fails to produce the clumps.

We now solve the balance Eq. (6). The collisional lifetime of resonant particles against mutual collisions, Eq. (19), depends on $n$, which makes the whole equation Eq. (6) quadratic in $n$. At $t \rightarrow \infty$, the total number of resonant particles tends to

$n=\frac{\hat{T}_{\text {coll }}}{2}\left[\sqrt{\frac{4 \dot{n}^{+} p_{\text {res }}}{\hat{T}_{\text {coll }}}+\left(\frac{1}{T_{\text {res }}}+\frac{1}{T_{\text {coll }}^{0}}\right)^{2}}-\left(\frac{1}{T_{\text {res }}}+\frac{1}{T_{\text {coll }}^{0}}\right)\right]$. 


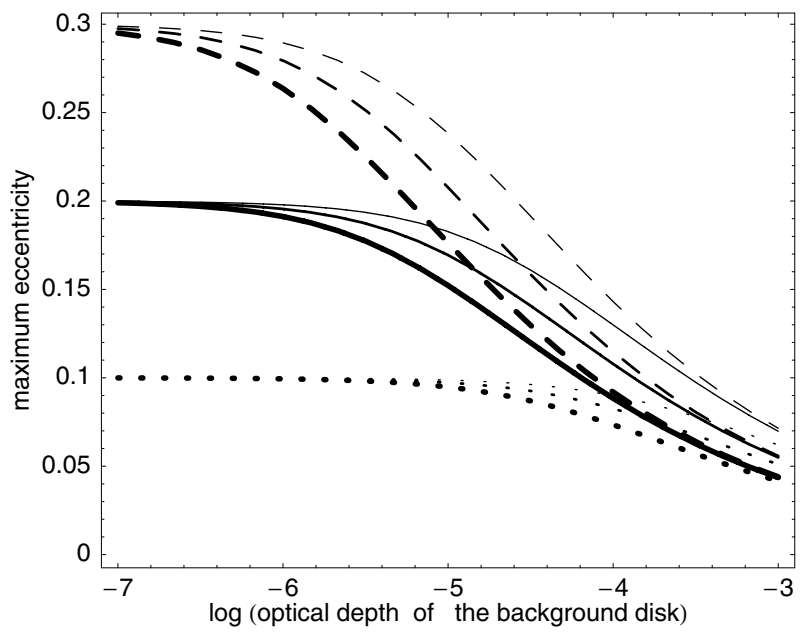

Fig. 3. Maximum orbital eccentricity of the clump particles as a function of the optical depth of the "regular" disk $\tau_{0}$. Dashed, solid, and dotted lines: $e_{\text {res }}=0.3,0.2$, and 0.1 , respectively. Thick, medium, and thin lines correspond to $\beta=0.05,0.10$, and 0.20 .

In the limit $n \ll n_{0}$, meaning relatively faint clumps and a relatively bright "regular" disk, the fourth term of Eq. (6) is much smaller than the third one, so that Eq. (6) is linear in $n$. In this case, the above solution simplifies to

$n=\frac{\dot{n}^{+} p_{\text {res }}}{T_{\text {res }}^{-1}+\left(T_{\text {coll }}^{0}\right)^{-1}}$.

It remains to estimate the optical depth of the resonant clumps. The clumps are not expected to be uniform for several reasons. First, even if the orbital eccentricities of all grains were equal, the second Kepler's law and the synodic motion of the particles relative to the planet would make the surface density of the resonant population non-uniform. Second, the resonant population consists of grains with a distribution of eccentricities, which is not uniform either: orbital dynamics forces the particle to spend most of the time in orbits with higher eccentricities up to $e_{\text {res }}$ (see Eq. (4)), whereas mutual collisions tend to eliminate these long-lived grains, shifting the distribution towards $e \ll e_{\text {res }}$. A superposition of these effects makes a spatial distribution of the optical depth in the clumps rather complex and dissimilar for different resonances and libration amplitudes $A$.

To compute the normal optical depth $\tau$ of the clumps, we use the following approximate method. We multiply $n$ by the crosssection area of a particle, $\pi s^{2}$, and divide the result by an approximate area of the clumps $S$. Noting that the area of the annulus is $S_{0}=4 \pi a^{2} e_{\max }$, we introduce the fractional area occupied by the clumps, $\hat{S} \equiv S / S_{0}$. Then,

$\tau \approx \frac{n s^{2}}{4 \hat{S} a^{2} e_{\max }}$.

The fractional area $\hat{S}$ was determined with the aid of a straightforward numerical simulation, by directly counting the points on the scatter plots. We considered the 3:2 resonance again, assuming different values of $e_{\max }$ between zero and $e_{\text {res }}=0.2$, as well as different values of $A$. For $e_{\max }=0.2$, taking into account all spatial locations reached by the particles locked in the resonance leads to $\hat{S}$ from $\approx 0.5$ for $A=20^{\circ}$ to $\approx 0.8$ for $A=90^{\circ}$. Defining, however, the regions with $30 \%$ of the peak brightness as a clump boundary, we arrive at values $\approx 0.1$ for $A=20^{\circ}$ to $\approx 0.3$ for $A=90^{\circ}$. Dependence on $e_{\max }$ turned out to be rather
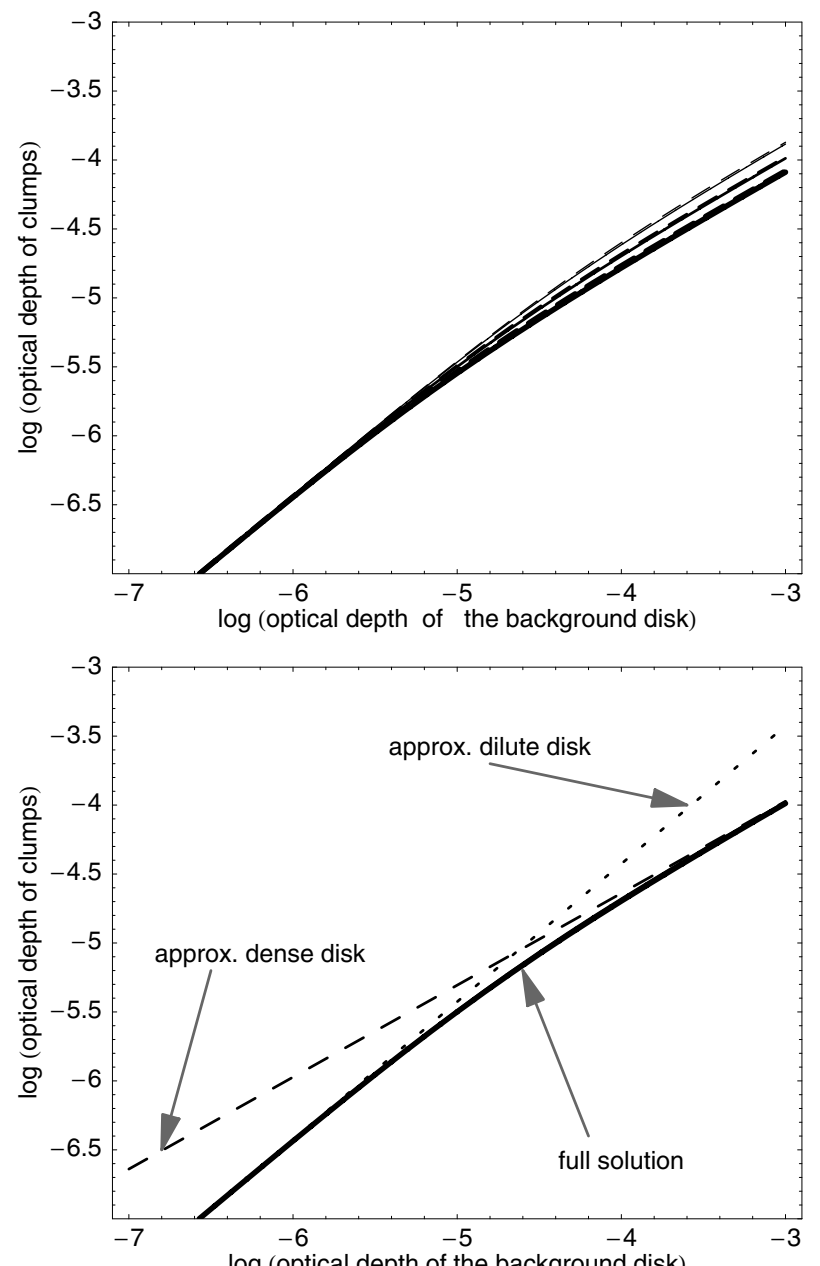

$\log$ (optical depth of the background disk)

Fig. 4. Normal optical depth of the resonant clumps $\tau$ as a function of the optical depth of the "regular" disk $\tau_{0}$. Top: full solution (20) of the balance equation (solid lines) and solution (21) of the linearized balance equation (dashed lines). Thick, medium, and thin lines correspond to $\beta=0.05,0.10$, and 0.20 , respectively. Bottom: full solution (20) of the balance equation for $\beta=0.1$ (solid line) versus the tenuous disk approximation (27) (dotted) and denser disk approximation (29) (dashed).

weak: $\hat{S}$ grows slightly with decreasing $e_{\max }$. We therefore adopt a simple $\hat{S}=0.2$ in our numerical examples, and stress that the actual $\tau$ in the brightest portions of the clumps is several times larger than we predict.

Figure 4 (top) depicts the resulting optical depth of the resonant blobs versus optical depth of the "regular" disk. The optical depth of clumps grows more slowly than that of the non-resonant population. A comparison between solid lines (Eq. (20)) and dashed ones (Eq. (21)) of the same thickness shows that the linear approximation (21) provides very good accuracy.

We now seek an approximate analytic scaling formula for the optical depth of the clumps, considering the stellar mass $M_{*}$, the planet's orbital radius $a_{\mathrm{p}}$, and a number of other quantities as free parameters. The ratio of the normal optical depth of the clumps, $\tau$, and of the background disk, $\tau_{0}$, is

$\frac{\tau}{\tau_{0}}=\frac{n}{n_{0}} \cdot \frac{1-p_{\text {res }} / 2}{\hat{S}}$

From Eq. (9) and an obvious relation

$n=\dot{n}^{+} p_{\text {res }} T$, 
we obtain

$\frac{\tau}{\tau_{0}}=p_{\text {res }} \cdot \frac{T}{\hat{S} T_{\mathrm{PR}}}$

where $T_{\mathrm{PR}}$ is given by Eqs. (10) and (8).

For tenuous disks (such that $T_{\text {coll }} \gg T_{\text {res }}$ ), $T \approx T_{\text {res }}$ and $e_{\max }(T) \approx e_{\text {res }}$. Therefore,

$\frac{\tau}{\tau_{0}} \approx p_{\mathrm{res}} \cdot \frac{T_{\mathrm{res}}}{\hat{S} T_{\mathrm{PR}}}=p_{\mathrm{res}} \cdot \frac{B T_{\mathrm{res}}}{4 \hat{S} e_{\mathrm{res}}}$

or, with the aid of Eq. (13),

$\frac{\tau}{\tau_{0}}=p_{\mathrm{res}} \cdot \frac{p+q}{q} \cdot \frac{e_{\mathrm{res}}}{4 \hat{S}}$.

The same formula in a form that is convenient for numerical estimates is

$$
\frac{\tau}{\tau_{0}}=0.125\left(\frac{p_{\text {res }}}{0.5}\right)\left(\frac{\hat{S}}{0.2}\right)^{-1}\left(\frac{e_{\text {res }}}{0.2}\right)\left(\frac{p+q}{q}\right) .
$$

This equation shows that $\tau / \tau_{0}$ is typically smaller (but not much smaller) than unity. In some cases it can exceed unity, however.

For denser disks (such that $T_{\text {coll }} \ll T_{\text {res }}$ ), the lifetime $T$ in Eq. (25) has to be evaluated iteratively, which complicates the calculations considerably. To keep the treatment at a reasonably simple level, we therefore assume that $n \ll n_{0}$, which is justified by Eq. (23) and by the fact that $\tau$ does not exceed $\tau_{0}$ for sufficiently dusty disks. In this case $T \approx T_{\text {coll }}^{0}=\hat{T}_{\text {coll }} / n_{0}$. We then substitute into Eq. (25) expressions (19) for $\hat{T}_{\text {coll }},(9)$ for $n_{0},(10)$ for $T_{\mathrm{PR}}$, and so on "to the whole depth" to express them through the free parameters. Omitting straightforward, but lengthy algebra, the result is

$$
\begin{aligned}
\tau \approx & p_{\text {res }} \cdot \frac{1}{4 \hat{S}} \\
& \times\left[\frac{\beta \pi \sin \epsilon}{c\left(1-\frac{p_{\text {res }}}{2}\right)(1-\beta)^{2 / 3}} \sqrt{\frac{G M_{*}}{a_{\mathrm{p}}}} \tau_{0}^{2}\right. \\
& \left.\times\left(\frac{p+q}{q}\right)^{2}\left(\frac{p}{p+q}\right)^{1 / 3}\right]^{1 / 3},
\end{aligned}
$$

where the square-root expression is the planet's orbital velocity. Dropping factors in brackets that are close to unity, we obtain a somewhat cruder version of the same formula which is convenient for numerical estimates:

$$
\begin{aligned}
\tau \approx & 9 \times 10^{-6}\left(\frac{p_{\text {res }}}{0.5}\right)\left(\frac{\hat{S}}{0.2}\right)^{-1}\left(\frac{\beta}{0.1}\right)\left(\frac{\epsilon}{0.1 \mathrm{rad}}\right) \\
& \times\left(\frac{M_{*}}{M_{\odot}}\right)^{1 / 6}\left(\frac{a_{\mathrm{p}}}{100 \mathrm{AU}}\right)^{-1 / 6}\left(\frac{\tau_{0}}{10^{-4}}\right)^{2 / 3}\left(\frac{p+q}{q}\right)^{2 / 3} .
\end{aligned}
$$

The bottom panel in Fig. 4 compares both analytic approximations, Eqs. (27) and (29), with each other and with the "exact" solution (20).

Equations (27) and (29) explicitly show the dependence of $\tau$ on many parameters: the stellar mass $M_{*}$, the planet's orbital radius $a_{\mathrm{p}}$, the resonance integers $p$ and $q$, the typical $\beta$ value of constituent particles, and others. For tenuous disks, $\tau$ is nearly proportional to $\tau_{0}$. For denser disks, $\tau \propto \tau_{0}^{2 / 3}-\mathrm{a}$ "slowdown" effect seen before in Fig. 4. The clumps' optical depth is nearly independent of the planet's distance from the star $a_{\mathrm{p}}$ for tenuous disks and slightly decreases with increasing $a_{\mathrm{p}}$ for larger $\tau_{0}$. The dependence on the stellar mass $M_{\star}$ is absent for dilute disks and weak for denser ones: $\tau$ slightly increases for more massive primaries. Note that the last statement is only true if we fix $\beta$ and not $s$. If the grain radius $s$ were treated as a free parameter, we would have to express $\beta$ through $s$ in Eq. (29), which would yield an additional $M_{\star}^{1 / 3}$ in Eq. (29), making the dependence on the stellar mass stronger. Next, an important dependence on the planet mass $M_{\mathrm{p}}$ is implicit (through the capture probability $p_{\text {res }}$ ); we will discuss it later. The fractional area of the clumps, $\hat{S}$, depends on the maximum orbital eccentricity of the resonant particles, and thus on other parameters such as $\tau_{0}$, but only weakly. For denser disks, $\tau$ increases, albeit only slightly, with the semi-opening angle of the background disk.

Figure 5 presents the "exact" solution, based on Eq. (20), in the form of contour plots of equal $\tau$, the average optical depth of the clumps. The figure shows that the phenomenon seen in Fig. 4 - "slowdown" of the growth of $\tau$ at higher $\tau_{0}$ - holds for all distances from the star $a_{\mathrm{p}}$. The effect is a bit more pronounced at larger $a_{\mathrm{p}}$. A comparison of the left and right panels demonstrates another effect: for given $\tau_{0}$ and $a_{\mathrm{p}}$, disks around more massive/luminous stars can develop somewhat denser clumps. All these effects are almost negligible, however.

As for any analytic model, our model rests on a number of simplifying assumptions, which we now discuss. One of those is a constant value of the resonance trapping probability $p_{\text {res }}$, which we have set to 0.5 in the numerical examples. In fact, it is through this quantity that the planet mass $M_{\mathrm{p}}$ would enter the model. This probability also depends on a particle's $\beta$, orbital semimajor axis $a$, and it varies from one resonance to another. Non-zero inclinations can affect the trapping probability as well. To the best of our knowledge, analytic or empirical formulas for $p_{\text {res }}$ as a function of $M_{\mathrm{p}}$ are only available for some subspaces of these parameters, and only for the planar problem (Beaugé \& Ferraz-Mello 1994; Lazzaro et al. 1994). It is clear, however, that resonance trapping is only possible within a certain planet mass range. If the planet is too massive, the orbits of dust grains become chaotic (Kuchner \& Holman 2003). For a solarmass star, the maximum mass is typically several Jupiter masses. If, conversely, the planet mass is too low, the particles go through the resonance without getting trapped. Beaugé \& Ferraz-Mello (1994) investigated the problem analytically and found, for instance, $0.04 M_{\mathrm{J}}\left(M_{\mathrm{J}}=\right.$ Jupiter mass $)$ to be a minimum mass to trap grains with $\beta=0.1$ in a 3:2 MMR. In a more general study, Lazzaro et al. (1994) found that for a planet with $M_{\mathrm{p}} \gtrsim 0.01 M_{\mathrm{J}}$ and for micrometer-sized particles with eccentricities lower than a few percent, there are large regions of capture in phase diagrams. These analytic estimates are supported by direct numerical integrations. Deller \& Maddison (2005), for instance, found a high efficiency of capturing for a wide range of planet masses, from $0.01 M_{\mathrm{J}}$ to $3 M_{\mathrm{J}}$, assuming a solar-mass central star. Within these limits $p_{\text {res }}$ shows rather a weak dependence on $M_{\mathrm{p}}$, namely a moderate growth with $M_{\mathrm{p}} / M_{*}$ (Beaugé \& Ferraz-Mello 1994; Lazzaro et al. 1994), justifying our simple assumption $p_{\text {res }}=0.5$.

Apart from a fixed $p_{\text {res }}$, we used a fixed value $e_{\text {res }}$ of 0.2 in the numerical examples. Contrary to $p_{\text {res }}$, this quantity is not important at all for denser disks, because the maximum eccentricity attained by the grains is limited by collisions, rather than by resonant dynamics; see Fig. 3 . That is why $e_{\text {res }}$ does not enter Eqs. (29) and (30). For low optical depths, $\tau$ is directly proportional to $e_{\text {res }}$, see Eqs. (27) and (28). As $e_{\text {res }}$ is known to lie approximately in the range $[0.1,0.4]$, the uncertainty is only a factor of two even in this case. 

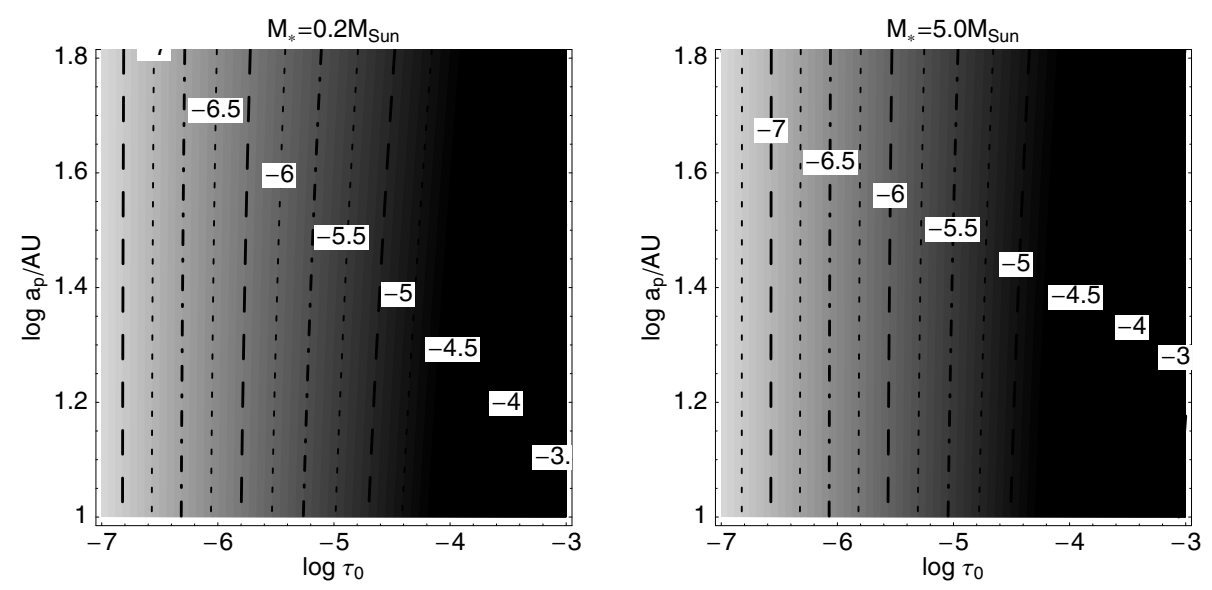

Fig. 5. Contour plots of the normal optical depth of the resonant clumps $\tau$, as a function of the optical depth of the "regular" disk $\tau_{0}$ and the planet's distance from the star. Left and right: stars with 0.2 and $5.0 M_{\odot}$. Contours are labeled with the values of $\log \tau$. Assumed $\beta=0.10$.

In the above treatment, we assumed destructive collisions to simply eliminate both colliders. This is supported by simple estimates. For two colliders of equal size $s$, the minimum relative speed $v$ needed to disrupt and disperse them, is determined by (see Eq. (5.2) of Krivov et al. 2005)

$v_{\min }=\sqrt{8 Q_{\mathrm{D}}^{*}}$,

where $Q_{\mathrm{D}}^{*}$ is the minimum specific energy for fragmentation and dispersal. At dust sizes, $Q_{\mathrm{D}}^{*} \sim 10^{7}$ to $10^{8} \mathrm{erg} \mathrm{g}^{-1}$ (e.g. Grigorieva et al. 2007). This gives $v_{\text {min }} \sim 100$ to $300 \mathrm{~m} \mathrm{~s}^{-1}$, much smaller than typical relative velocities (17). Under the same conditions, the largest fragment is an order of magnitude smaller than either collider (see Sect. 5.3 of Krivov et al. 2005), which allows us to ignore the generated fragments. We also ignore cratering collisions that may only moderately affect the mass budget, the timescales, and the optical depth of the clumps.

Perhaps the most fundamental simplification that we make is to choose a single particle size. This leads, in particular, to overestimation of $T_{\text {coll }}^{0}$ and $T_{\text {coll }}$. The actual collisional lifetime will be shorter, because the grains that dominate the clumps will preferentially be destroyed by somewhat smaller, hence more abundant, particles. Collisions with interstellar grains may shorten the collisional lifetime even more.

In the future, it would be interesting to develop a more elaborate kinetic model with $n$ as a function of size $s$ (Krivov et al. $2005,2006)$. Such a model would be particularly interesting for dilute disks with $\tau_{0} \lesssim 10^{-6}$, expected to be resolved with future instruments (Herschel, ALMA, JWST, DARWIN, SAFIR, etc.)

\section{Scenario II}

In this section, we analyze the alternative scenario II, in which dust parent bodies are already locked in a certain resonance, so that the dust they produce resides in the same resonance, creating the clumps.

This scenario is more difficult to quantify than the first one, for the following reasons. In scenario I, the dust production mechanisms were "hidden" in one quantity, the dust injection rate $\dot{n}^{+}$, which we estimated from an "observed" value, optical depth $\tau_{0}$. This way essentially eliminates much of the uncertainty in modeling scenario I. In contrast, a physical description of scenario II requires knowledge of various parameters of resonant planetesimal populations. These are not observable directly and can only be accessed by extensive modeling, taking into account the wealth of processes that drive a forming planetary system (Wyatt 2003). Even if the parameters of a planetesimal ensemble were known, another major source of uncertainties would be the necessity of modeling a collisional cascade from planetesimal-sized down to dust-sized objects, i.e. across some 30 orders of magnitude in mass. Therefore, we construct here the simplest possible model to explore the efficiency of scenario II, while being aware of its roughness.

Consider a population of planetesimals with the total mass $M$, locked in the $(p+q): p$-resonance. These planetesimals occasionally experience destructive collisions, in which smaller fragments are produced. Since the relative velocities of fragments are small compared to orbital velocities, most of them cannot leave the resonance and stay in the system. Gradual loss of material occurs through dust-sized fragments: below a certain minimum radius $s_{\min }$, the radiation pressure will force the particles out of the resonance (Wyatt 2006). Relative velocities, expected to be higher for smaller fragments, will also liberate a fraction of particles from the resonance.

We numerically investigated the conditions under which the fragments remain captured in the resonance. Again, we took the $\epsilon$ Eri system with $M_{*}=0.8 M_{\odot}, M_{\mathrm{p}}=0.1 M_{\mathrm{J}}, a_{\mathrm{p}}=40 \mathrm{AU}$. We first chose the set of planetesimal orbits, all with the resonant (3:2) value of the semimajor axis, randomly chosen eccentricities between 0 and 0.1 , inclinations between 0 and $0.1 \mathrm{rad}$, and three angular elements between $0^{\circ}$ and $360^{\circ}$, and selected those that turned out to be locked in resonance with libration amplitudes not larger than $30^{\circ}$ (Fig. 6 top left). From the same set of planetesimals, we then released dust particles with different $s$ (and $\beta$ ) and different velocity increments $\boldsymbol{u}$ relative to the parent bodies and numerically followed the motion of these ejecta to find out whether they will stay in the resonance as well. As a criterion for staying in the resonance, we simply require that the resonant argument $\Phi$ librates with a moderate amplitude $A$, not exceeding 30-50 degrees. We thus exclude shallow resonances that can easily be destroyed by external perturbations and, besides, lead to nearly axisymmetric ring-like configurations.

The results are presented in Fig. 6 in the form of phase portraits of eccentricity and resonant angle, in polar coordinates. It is seen that particles larger than about $40 \mu \mathrm{m}$ (or $\beta<0.003$ ), if ejected with relative velocities $u<10 \mathrm{~m} \mathrm{~s}^{-1}$, typically stay locked in the resonance. Thus $\beta_{\text {crit }} \approx 0.003$ and $u_{\text {crit }} / v_{\mathrm{k}} \approx$ 0.003 . The fact that $\beta_{\text {crit }}$ and $u_{\text {crit }} / v_{\mathrm{k}}$ are close to each other is not surprising. Both radiation pressure and an initial velocity "kick" do nothing else than cause the initial semimajor axis and 

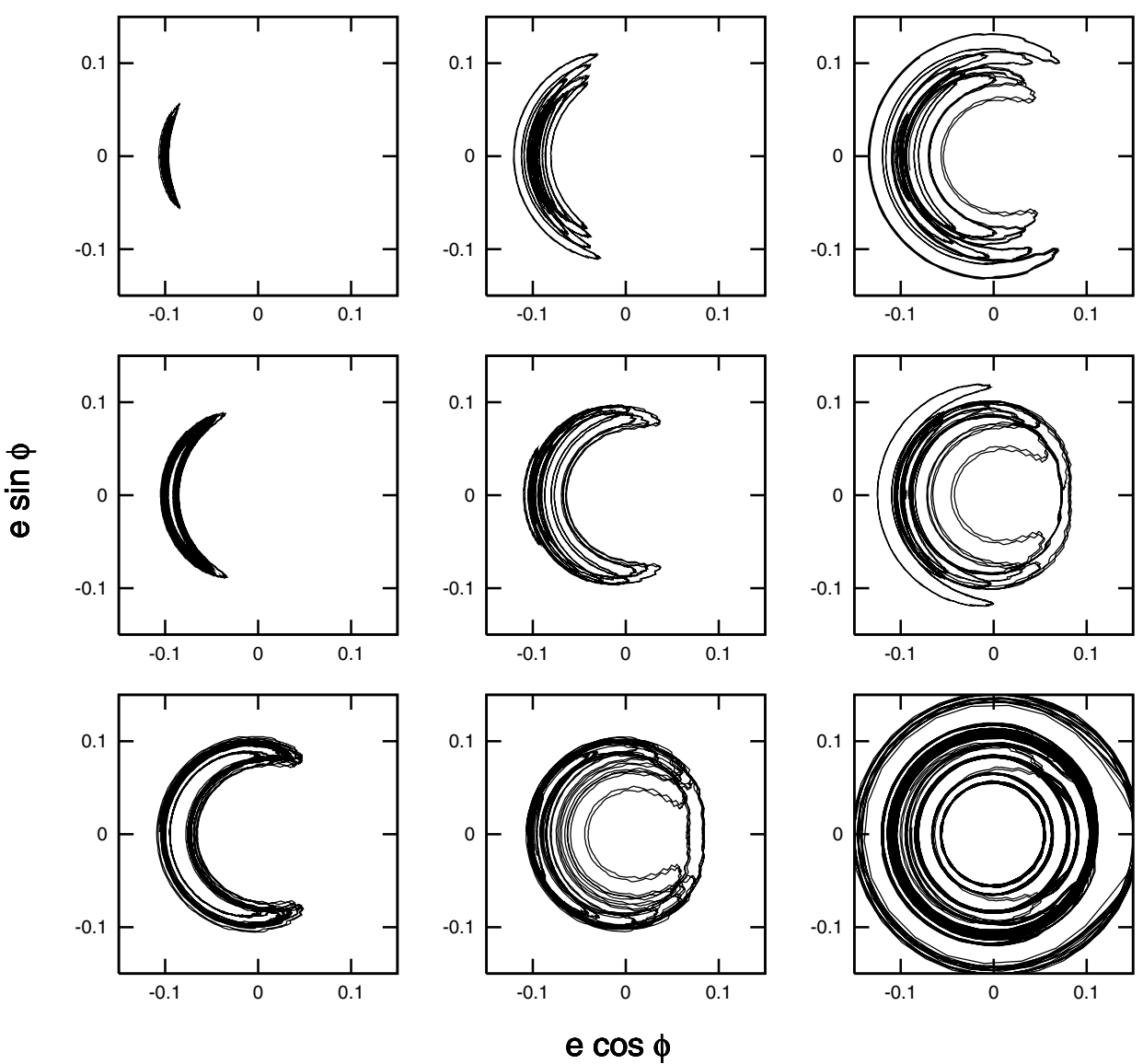

Fig. 6. Phase portraits of resonant planetesimals and the dust grains they release in mutual collisions. Top: planetesimals themselves, middle: $40 \mu \mathrm{m}$-sized dust, bottom: $20 \mu \mathrm{m}$-sized dust. From left to right: the relative velocities of 0,10 , and $20 \mathrm{~m} \mathrm{~s}^{-1}$.

eccentricity of the particle orbit to differ from those of a parent planetesimal. Within a factor of several, $\Delta a / a \sim e \sim \beta$ in the first case and $\Delta a / a \sim e \sim u / v_{\mathrm{k}}$ in the second. Thus our finding means that a maximum relative change in $(a, e)$ that still leaves the grains in the resonance is several tenths of a percent.

Interestingly, a similar problem was investigated before in quite a different context. Krivov \& Banaszkiewicz (2001) studied the fate of tiny icy ejecta from the saturnian moon Hyperion, locked in the 4:3-MMR with Titan and found that about a half of $100 \mu \mathrm{m}$-sized grains remained locked in the resonance.

One of the two effects - the $\beta$-threshold - was recently considered in great detail by Wyatt (2006). For the 3:2-resonance, he found

$\beta_{\text {crit }}=0.034\left(\frac{M_{\mathrm{p}} / M_{\mathrm{J}}}{M_{*} / M_{\odot}}\right)^{1 / 2}$.

For $M_{*}=0.8 M_{\odot}$ and $M_{\mathrm{p}}=0.1 M_{\mathrm{J}}$, this gives $\beta_{\text {crit }}=0.012$, which is a slightly more "tolerant" a threshold than the one we observed in our simple simulation.

As our numerical test was done for one pair $\left(M_{*}, M_{\mathrm{p}}\right)$, it still does not allow us to judge how the threshold of relative velocity scales with stellar mass and planetary mass. To find an approximate scaling rule, we therefore undertook a series of numerical integrations with 5 values of $M_{\mathrm{p}}(0.03,0.1,0.3$, 1.0 , and $\left.3.0 M_{\mathrm{J}}\right)$ and 6 values of $M_{*}(0.1,0.2,0.5,1.0,2.0$, and $\left.5.0 M_{\odot}\right), 5 \times 6=30$ runs in total. In each run, we integrated trajectories of 100 particles $\beta=0$ released with different velocities from planetesimals locked in the resonance with libration amplitudes not larger than $30^{\circ}$. For each run separately, we determined the mean velocity threshold below which the particles stay in the resonance, having libration amplitudes of not more than $30^{\circ}, 40^{\circ}$, and $50^{\circ}$ (Fig. 7, symbols). We then fitted the results with a power law

$u_{\text {crit }} / v_{\mathrm{k}}=\mathcal{A}\left(\frac{M_{\mathrm{p}} / M_{\mathrm{J}}}{M_{*} / M_{\odot}}\right)^{\mathcal{B}}$.

The constants are $\mathcal{A} \approx 0.007( \pm 40 \%)$ and $\mathcal{B} \approx 0.28( \pm 10 \%)$ for the "threshold" $A$ of $30^{\circ}$. The fits are shown in Fig. 7 with lines. For the $\epsilon$ Eri under the same assumptions as above, Eq. (33) gives $u_{\text {crit }}=14 \mathrm{~m} \mathrm{~s}^{-1}$ for a planet with mass $M_{\mathrm{p}}=0.1 M_{\mathrm{J}}$ and $26 \mathrm{~m} \mathrm{~s}^{-1}$ for $M_{\mathrm{p}}=1.0 M_{\mathrm{J}}$.

We note that the dust grains making the observed clumps have gone through a collisional cascade. The cascade starts with collisions of the largest planetesimals with mass $m_{\max }$ (or radius $s_{\max }$ ), followed by collisions of progressively smaller bodies, until dust grains with mass $m_{\min }$ (corresponds to $s_{\min }$ or $\beta_{\text {crit }}$ explained above) are produced. We first consider a single collision and estimate the fraction of material which has $u<u_{\text {crit }}$. It can be approximated as (Nakamura \& Fujiwara 1991)

$\Psi\left(<u_{\text {crit }}\right)=1-\left(u_{0} / u_{\text {crit }}\right)^{\gamma} \quad\left(u_{\text {crit }} \geq u_{0}\right)$

where laboratory impact experiments suggest the lower cutoff $u_{0} \sim 5 \mathrm{~m} \mathrm{~s}^{-1}$ (for dust sizes) and the exponent $\gamma \sim 2$ (for consolidated material, such as low-temperature ice). Of course, the power-law fit (34) should be treated with great caution. In reality, no sharp lower cutoff exists, so that $u_{0}$ just places the lower bound on the speed range in which Eq. (34) is valid. A single power-law index $\gamma$ holds only in a limited velocity range. 


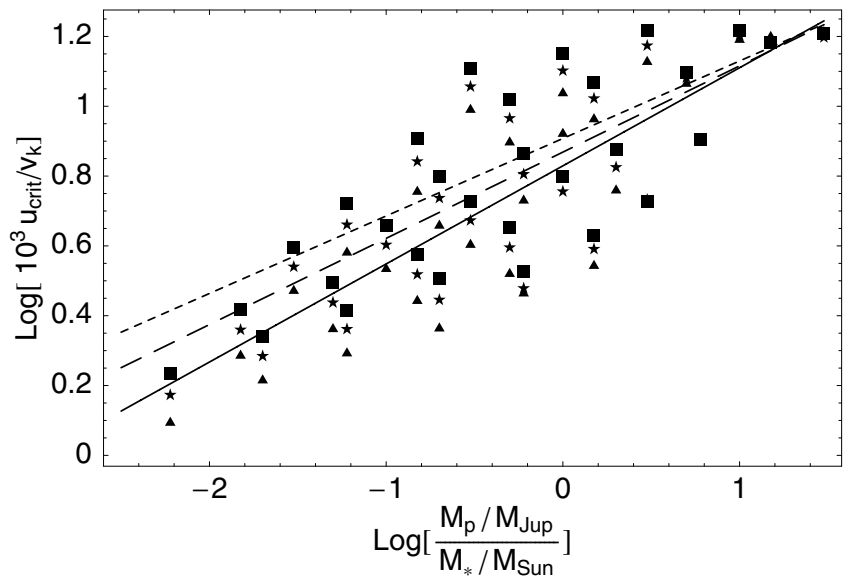

Fig. 7. Critical relative velocity of fragments $u_{\text {crit }} / v_{\mathrm{k}}$ to stay in the resonance, as a function of the planet-to-star mass ratio. Symbols: results of individual runs; lines: power law fits (33). Different maximum $A$ of particles were adopted as a criterion of staying in the resonance: $A=30^{\circ}$ (triangles and solid line), $A=40^{\circ}$ (squares and dashed line), $A=50^{\circ}$ (stars and dotted line).

Both $u_{0}$ and $\gamma$ may vary significantly for different materials, morphology of the colliders, impact velocities, incident angles, target and projectile masses, and other parameters of collision (e.g. Fujiwara 1986).

According to Eq. (34), most of the fragments of a single collision have velocities slightly above $u_{0}$. This is consistent with many impact experiments that reported relative velocities in the range 5-20 $\mathrm{m} \mathrm{s}^{-1}$ as typical (e.g. Arakawa et al. 1995; Giblin et al. 1998). These typical velocities - and therefore the cutoff value $u_{0}$ - depend on the fragments' mass. Indeed, Nakamura \& Fujiwara (1991) found a mass-velocity relation $u \propto m^{-1 / 6}$ to fit their experimental data. Since all impact experiments are only possible and have only been done for the lower end of the size distribution (objects less than one meter in size), we will assume that $u_{0} \sim 5 \mathrm{~m} \mathrm{~s}^{-1}$ is valid for "the last" collisions in the cascade and that $u_{0}$ diminishes according to the $m^{-1 / 6}$ law for "previous" collisions of larger objects. Needless to say, this prescription should also be treated with caution and is only suitable for crude estimates of the velocity-related effects we are aiming at.

A natural question is: what is the average number of collisions, $k$, needed to produce a dust particle with $s_{\min }=30 \mu \mathrm{m}$ out of parent planetesimals with size $s_{\max }=10 \mathrm{~km}$ ? Simple analytical calculations done in the Appendix yield $k \approx 8$. This result depends, albeit rather weakly, on several parameters, such as the mass of the largest fragment in a single binary collision.

We now estimate roughly the loss of particles due to $u>u_{\text {crit }}$ over the whole cascade. We simply replace the real cascade, in which each collision generates a mass distribution of fragments, with a sequence of $k=8$ steps. In the first step, planetesimals of mass $m_{\max }$ collide with each other and produce smaller fragments of equal mass. In the next step, the latter collide with each other, and so on, until the fragments of mass $m_{\min }$ are produced. As follows from Eq. (34) and the mass-velocity relation discussed above, the fraction of dust mass that is able to stay in the resonance can be estimated as

$$
\begin{aligned}
\Psi_{\text {cascade }} \approx & {\left[1-\left(\frac{u_{0}}{u_{\text {crit }}}\right)^{\gamma}\right] \times\left[1-\left(\frac{u_{0} \delta^{1 / 6}}{u_{\text {crit }}}\right)^{\gamma}\right] } \\
& \times \ldots \times\left[1-\left(\frac{u_{0} \delta^{k / 6}}{u_{\text {crit }}}\right)^{\gamma}\right],
\end{aligned}
$$

where

$\delta \equiv\left(\frac{m_{\min }}{m_{\max }}\right)^{1 / k} \ll 1$.

In nearly all situations, only the first term matters, which means that most of the material is lost to the "velocity effect" at the last collision. In what follows, we thus adopt

$\Psi_{\text {cascade }} \approx 1-\left(\frac{u_{0}}{u_{\text {crit }}}\right)^{\gamma}$.

On the whole, the resonant population represents a nearly closed system, losing material only slowly through the smallest and fastest grains, which reduces the mass of the population on extremely long (typically Gyr) timescales. This makes it possible to apply Dohnanyi's (1969) theory of a closed collisional system. Provided that the critical impact energy for fragmentation and dispersal $Q_{\mathrm{D}}^{*}$ is independent of size over the size regime considered, he has shown that such a system evolves to a steady state, in which the mass distribution is a simple power law: $n(m) \propto m^{-1-\alpha}$ with $\alpha=5 / 6=0.83$. Durda \& Dermott (1997) generalized this result by considering a more realistic, size-dependent $Q_{\mathrm{D}}^{*}$. They have shown that a power-law dependence of $Q_{\mathrm{D}}^{*}$ on size preserves a power-law size distribution, but makes it steeper or flatter, depending on whether $Q_{\mathrm{D}}^{*}$ decreases or increases with size. For $Q_{\mathrm{D}}^{*} \propto s^{-0.3}$, which is a plausible choice in the strength regime (see Krivov et al. 2005, and references therein), Durda and Dermott found $\alpha=0.87$.

The total cross section of the steady-state population is

$\Sigma=\frac{1-\alpha}{\alpha-2 / 3} \pi^{1 / 3}\left(\frac{3}{4 \rho}\right)^{2 / 3} \frac{M}{m_{\max }^{1-\alpha} m_{\min }^{\alpha-2 / 3}} \Psi_{\text {cascade }}$,

where $\rho$ is the material density of the objects. For $\alpha=5 / 6$, Eq. (38) takes a symmetric form

$\Sigma=\pi^{1 / 3}\left(\frac{3}{4 \rho}\right)^{2 / 3} \frac{M}{m_{\min }^{1 / 6} m_{\max }^{1 / 6}} \Psi_{\text {cascade }}$

Equations (38) and (39) show that the results depend on $m_{\min }$ and $m_{\max }$ only weakly.

Like in the first scenario, we then divide $\Sigma$ by the area of the clumps, $S=4 \pi \hat{S} a^{2} e_{\max }$, to obtain an estimate of the clumps' normal optical depth. The results are presented in Fig. 8 for the $\epsilon$ Eri case. We assumed again $M_{*}=0.8 M_{\odot}, \epsilon=0.1=6^{\circ}$, and considered the 3:2 resonance (planetesimals with $a=56 \mathrm{AU}$ ) with a planet of mass $M_{\mathrm{p}}=0.1 M_{\mathrm{J}}$. We adopted $s_{\max }=10 \mathrm{~km}$, $e_{\max }=0.2$, and $\hat{S}=0.2$. From (32), $\beta_{\text {crit }}=0.012$ (or $s_{\min }=$ $12 \mu \mathrm{m})$. As in Fig. 4, we plot the normal optical depth of the clumps $\tau$, but now as a function of the planetesimal population mass $M$. Two size-distribution indexes were used: Dohnanyi's $\alpha=0.833$ and a more realistic $\alpha=0.87$. Our best-guess choice, $\alpha=0.87$ shows that the population with $M=0.01 M_{\oplus}\left(M_{\oplus}\right.$ is the Earth mass) would produce clumps with $\tau \sim 10^{-5}$. The same figure demonstrates that the dependence on $\gamma$ is moderate, but that on the cutoff value $u_{0}$ is rather substantial. We speculate that uncertainties in the fragment velocity distribution may introduce at least an order of magnitude, perhaps a larger, uncertainty in the resulting optical depth of the clumps.

Similar to the first scenario, we now investigate the dependence of the clump formation efficiency on various parameters. We use Eq. (38) with $\alpha=0.87$ and $\rho=2 \mathrm{~g} \mathrm{~cm}^{-3}$ and express $m_{\min }$ through $\beta_{\text {crit }}$ and then through $M_{*}$ and $M_{\mathrm{p}}$ by Eq. (32). 


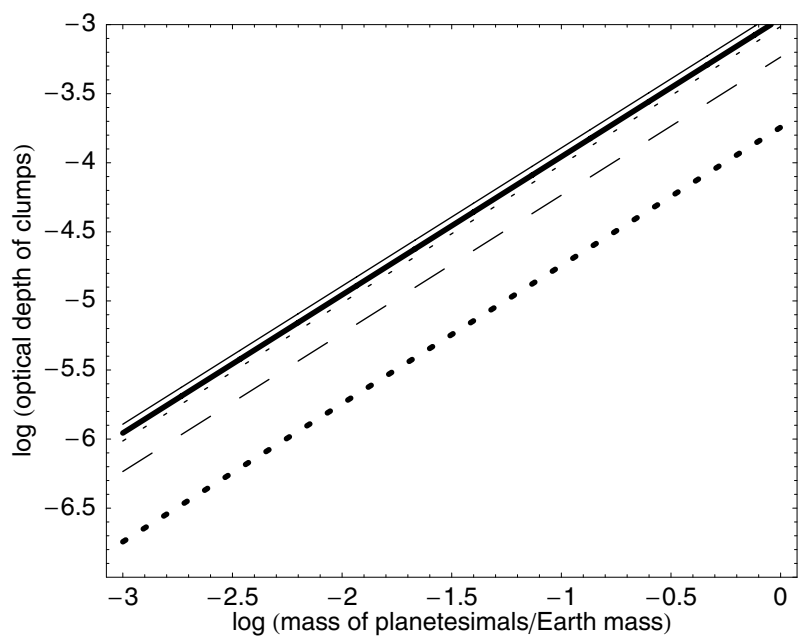

Fig. 8. Normal optical depth of the resonant clumps $\tau$ in scenario II, as a function of the planetesimal population mass $M$. Thick lines are for $u_{0}=5 \mathrm{~m} \mathrm{~s}^{-1}$ and $\gamma=2$ and different $\alpha$ : Dohnanyi's $\alpha=0.833$ (dashed) and a more realistic $\alpha=0.87$ (solid). Thin lines are for $\alpha=0.87$ and different $u_{0}$ and $\gamma: u_{0}=1 \mathrm{~m} \mathrm{~s}^{-1}$ and $\gamma=2$ (solid), $u_{0}=10 \mathrm{~m} \mathrm{~s}^{-1}$ and $\gamma=2$ (dashed), $u_{0}=5 \mathrm{~m} \mathrm{~s}^{-1}$ and $\gamma=1.5$ (dotted). Cf. Fig. 4 for scenario I.

By setting $\Psi_{\text {cascade }}$ to unity, we obtain a rough upper limit on the optical depth of the clumps:

$$
\begin{aligned}
\tau \lesssim & 4.3 \times 10^{-5}\left(\frac{\hat{S}}{0.2}\right)^{-1}\left(\frac{e_{\max }}{0.2}\right)^{-1} \\
& \times\left(\frac{s_{\max }}{10 \mathrm{~km}}\right)^{-0.39}\left(\frac{M}{0.1 M_{\oplus}}\right) \\
& \times\left(\frac{M_{\mathrm{p}}}{M_{\mathrm{J}}}\right)^{0.31}\left(\frac{M_{*}}{M_{\odot}}\right)^{-2.14}\left(\frac{a_{\mathrm{p}}}{100 \mathrm{AU}}\right)^{-2}\left(\frac{p}{p+q}\right)^{4 / 3}
\end{aligned}
$$

Each panel in Fig. 9 presents the results in form of the contour plots of equal $\tau$, the average optical depth of the clumps, on the total mass of the resonant planetesimals $M$ and planet's orbital radius $a_{\mathrm{p}}$, showing that $\tau$ grows with increasing $M$ and decreasing $a_{\mathrm{p}}$. Different panels illustrate the dependence on $\alpha, M_{*}$, and $M_{\mathrm{p}}$. As already seen, the dependence on $\alpha$ is quite strong. The dependence on the stellar mass is strong, too. However, in contrast to the first scenario, stars with higher luminosity produce less pronounced clumps. Dependence on the planet mass $M_{\mathrm{p}}$ is stronger than in the first scenario. More massive planets create brighter clumps.

Remember that all our estimates imply that the resonant population is in a steady state. Transient fluctuations in the population can be produced by recent major collisional events (Wyatt \& Dent 2002; Kenyon \& Bromley 2005; Grigorieva et al. 2007). Usually, the dust debris cloud generated by a "supercollision" of two large planetesimals is spread by the Keplerian shear into a relatively homogeneous ring in only $10^{4}$ to $10^{5}$ years (Kenyon $\&$ Bromley 2005). However, in a resonant population, most of the fragments should stay in the resonance and contribute to the clump brightness - for the reasons discussed above. The same Eqs. (38) and (40) apply, with $M$ now the total mass of both colliders. Roughly, a collision of two lunar-sized objects would generate $0.01 M_{\oplus}$ of debris, enough to produce well-observable clumps.

\section{Conclusions and discussion}

In this paper, we address the possible origin of azimuthal substructure in the form of clumps or blobs, observed in some debris disks such as $\epsilon$ Eridani. Two scenarios are considered. In the first case, the one discussed most in the literature, dust particles are produced in planetesimal belts exterior to resonance locations, brought to exterior planetary resonances by the P-R effect, and their resonance trapping leads to the formation of clumps. In the second case, dust-producing planetesimals are already locked in a resonance, for instance, as a result of planetary migration in the past, and the dust they produce resides in the same resonance, creating the clumps. For both scenarios, we have constructed simple analytic models in order to explore their applicability limits and efficiency for a wide range of stars, planets, disks, and planetesimal families.

1. Scenario I. We conclude that the efficiency of the first scenario strongly depends on the normal geometrical optical depth $\tau_{0}$ of the "regular" disk, from which dust is supplied to the clumps. The optical depth is known from observations. Besides, the brightness of the resulting clumps is nearly proportional to the probability of trapping "visible" dust grains in the resonance, $p_{\text {res }}$. This parameter can be constrained relatively well, too, with the aid of numerical integrations for one or another particular system and a specified resonance.

In disks with roughly $\tau_{0} \lesssim 10^{-4}-10^{-5}$, the first scenario works well, creating pronounced clumps. Their optical depth $\tau$ is usually lower than, but can be comparable to or even exceed, the optical depth of the background disk. In other words, in dilute disks the clumps can be almost as bright as, and sometimes brighter than, the underlying disk in the same region. This is the case in the solar system's interplanetary dust cloud and the dust in the Kuiper belt with their $\tau_{0} \lesssim 10^{-7}$. Further, this will be the case for the debris disks at which future telescopes are aiming; examples are: the Herschel Space Observatory, the Atacama Large Millimeter Array (ALMA), the James Webb Space Telescope (JWST), the space infrared interferometer DARWIN, and the Spitzer's and Herschel's follow-on SAFIR. But it is not the case for debris disks of the other stars resolved so far. The disks currently observed and the clumps observed in them, all have optical depth $>10^{-5}$.

For higher optical depths $\tau_{0}, \tau$ can still be high enough, but collisions are "killing" the clumps in the following way. They shorten the lifetimes of grains captured in the resonance, which prevents them from developing higher orbital eccentricities. Instead, the trapped particles remain in orbits with low eccentricities. The resonant population will look like a narrow ring without azimuthal structure, not like clumps. The dustier the disk, the narrower the resulting ring (for planets in nearcircular orbits), and the less pronounced the azimuthal structure. For dustier disks, we find that the optical depth of the clumps has a tendency to increase when the responsible planet orbits closer to the star and has a higher mass. It also tends to grow with the luminosity of the central star. Increasing the opening angle of the dust disk has the same effect. However, all these dependencies are quite weak.

In the case of $\epsilon$ Eridani, which falls into the category of "dustier" disks, the first scenario does not seem to be consistent with the presence of several longitudinally confined "blobs".

2. Scenario II. The efficiency of the second mechanism is obviously proportional to the total mass of the resonant population of planetesimals, but is strongly affected by other parameters, too. The brightness of the clumps produced by the second scenario increases with the decreasing luminosity of the star, 

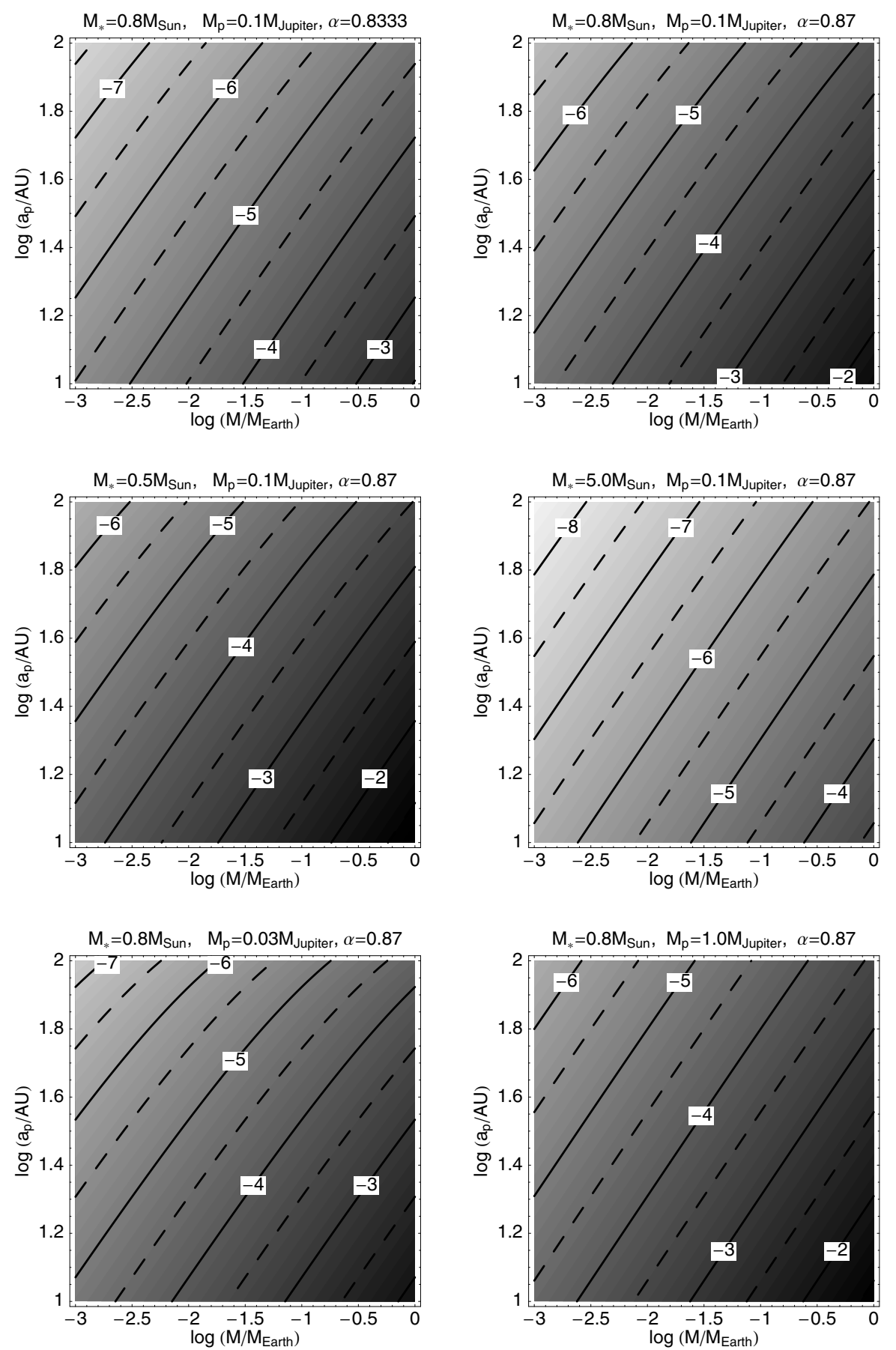

Fig. 9. Contour plots of the normal optical depth of the resonant clumps $\tau$ in scenario II, as a function of the planetesimal population mass $M$ and the planet's distance from the star. Top left and top right: Dohnanyi's $\alpha=0.833$ and more realistic $\alpha=0.87$, both for the same star with $0.8 M_{\odot}$ and a planet with 0.1 Jupiter mass. Middle left and middle right: stars with 0.5 and $5.0 M_{\odot}$, both for $\alpha=0.87$ and a planet with 0.1 Jupiter mass. Bottom left and bottom right: a planet with 0.03 Jupiter mass and 1 Jupiter mass, both for $\alpha=0.87$ and $M_{*}=0.8 M_{\odot}$. Contours are labeled with the values of $\log \tau$. Cf. Fig. 5 for scenario I.

increasing planetary mass, and decreasing orbital radius of the planet. All these dependencies are more pronounced than in the first scenario.

In the case of $\epsilon$ Eridani, a family with a total mass of $\sim 0.01$ to 0.1 Earth masses could well be sufficient to account for the observed optical depth of the clumps, roughly $\tau \sim 10^{-5}$. Thus this mechanism appears to be more relevant for systems like $\epsilon$ Eri.

It must be noted that the efficiency of scenario II is quite sensitive to the poorly known properties of the collisional grinding process. One is the critical energy for fragmentation and dispersal $Q_{\mathrm{D}}^{*}$ over the whole range of masses - from planetesimals down to dust - which strongly affects the equilibrium slope of the size distribution, and therefore the total cross section ares of the resonant clumps. Even more important is the distribution of the ejecta velocities in disruptive collisions, which determines what fraction of collisional debris is fast enough to leave the resonant population. For these reasons, models of the second scenario are quantitatively more uncertain than those of the first one.

Models presented here are the simplest possible ones we could construct. Accordingly, they involve a wealth of 
simplifying assumptions and therefore cannot substitute for developing more elaborate models. Yet we hope that our results can give useful guidelines and serve as a starting point for more detailed studies of clumps in debris disks.

Acknowledgements. We wish to thank Mark Wyatt and Jane Greaves for useful discussions and the anonymous referee for his/her thorough and very helpful review. We are grateful to Martin Reidemeister for his assistance with numerical runs of the integration routine. Miodrag Sremčević is supported by the Cassini UVIS project.

\section{References}

Arakawa, M., Maeno, N., Higa, M., Iijima, Y.-i., \& Kato, M. 1995, Icarus, 118, 341

Augereau, J.-C. 2004, in Extrasolar Planets: Today and Tomorrow, ASP Conf. Ser., 321, 305

Beaugé, C., \& Ferraz-Mello, S. 1994, Icarus, 110, 239

Burns, J. A., Lamy, P. L., \& Soter, S. 1979, Icarus, 40, 1

Deller, A. T., \& Maddison, S. T. 2005, ApJ, 625, 398

Dermott, S. F., Jayaraman, S., Xu, Y. L., Gustafson, B.Å. S., \& Liou, J.-C. 1994, Nature, 369, 719

Dohnanyi, J. S. 1969, J. Geophys. Res., 74, 2531

Durda, D. D., \& Dermott, S. F. 1997, Icarus, 130, 140

Fujiwara, A. 1986, Mem. Soc. Astron. It., 57, 47

Giblin, I., Martelli, G., Farinella, P., et al. 1998, Icarus, 134, 77

Gold, T. 1975, Icarus, 25, 489

Greaves, J. S., Holland, W. S., Moriarty-Schieven, G., et al. 1998, A\&A, 506, L133

Greaves, J. S., Holland, W. S., Wyatt, M. C., et al. 2005, ApJ, 619, L187

Grigorieva, A., Artymowicz, P., \& Thébault, P. 2007, A\&A, 461, 537

Holland, W. S., Greaves, J. S., Dent, W. R. F., et al. 2003, ApJ, 582, 1141

Holland, W. S., Greaves, J. S., Zuckerman, B., et al. 1998, Nature, 392, 788

Jackson, A. A., \& Zook, H. A. 1989, Nature, 337, 629

Kalas, P., Deltorn, J.-M., \& Larwood, J. 2001, ApJ, 553, 410
Kalas, P., Graham, J. R., Clampin, M. C., \& Fitzgerald, M. P. 2006, ApJ, 637, L57

Kenyon, S. J., \& Bromley, B. C. 2005, AJ, 130, 269

Krivov, A. V., \& Banaszkiewicz, M. 2001, Planet. Space Sci., 49, 1265

Krivov, A. V., Mann, I., \& Krivova, N. A. 2000, A\&A, 362, 1127

Krivov, A. V., Sremčević, M., \& Spahn, F. 2005, Icarus, 174, 105

Krivov, A. V., Löhne, T., \& Sremčević, M. 2006, A\&A, 455, 509

Kuchner, M. J., \& Holman, M. J. 2003, ApJ, 588, 1110

Kuchner, M. J., Reach, W. T., \& Brown, M. E. 2000, Icarus, 145, 44

Lagrange, A.-M., Backman, D. E., \& Artymowicz, P. 2000, in Protostars and Planets IV, ed. V. Mannings, A. P. Boss, \& S. S. Russell (Tucson: University of Arizona Press), 639

Lazzaro, D., Sicardy, B., Roques, F., \& Greenberg, R. 1994, Icarus, 108, 59

Lecavelier des Etangs, A., Scholl, H., Roques, F., Sicardy, B., \& Vidal-Madjar, A. 1996, Icarus, 123, 168

Liou, J.-C., \& Zook, H. A. 1997, Icarus, 128, 354

Liou, J.-C., \& Zook, H. A. 1999, AJ, 118, 580

Liou, J.-C., Zook, H. A., \& Dermott, S. F. 1996, Icarus, 124, 429

Liou, J.-C., Zook, H. A., Greaves, J. S., \& Holland, W. S. 2000, LPSC XXXI, LPI, Houston, TX, abstract No. 1416

Marzari, F., \& Vanzani, V. 1994, A\&A, 283, 275

Moro-Martín, A., \& Malhotra, R. 2005, ApJ, 633, 1150

Mouillet, D., Larwood, J. D., Papaloizou, J. B., \& Lagrange, A.-M. 1997, MNRAS, 292, 896

Nakamura, A., \& Fujiwara, A. 1991, Icarus, 92, 132

Ozernoy, L. M., Gorkavyi, N. N., Mather, J. C., \& Taidakova, T. A. 2000, ApJ, 537, L147

Quillen, A. C., \& Thorndike, S. 2002, ApJ, 578, L149

Reach, W. T., Franz, B. A., Weiland, J. L., et al. 1995, Nature, 374, 521

Thébault, P., Augereau, J. C., \& Beust, H. 2003, A\&A, 408, 775

Weidenschilling, S. J., \& Jackson, A. A. 1993, Icarus, 104, 244

Wilner, D. J., Holman, M. J., Kuchner, M. J., \& Ho, P. T. P. 2002, ApJ, 569, L115

Wyatt, M. C. 2003, ApJ, 598, 1321

Wyatt, M. C. 2006, ApJ, 639, 1153

Wyatt, M. C., \& Dent, W. R. F. 2002, MNRAS, 334, 589 
A. V. Krivov et al.: Clumps in debris disks, Online Material $p 1$

\section{Online Material}


A. V. Krivov et al.: Clumps in debris disks, Online Material p 2

\section{Appendix A: Number of collisions \\ over the collisional cascade}

Consider the collisional cascade that produces small grains of mass $m$ from parent bodies of mass $m_{\max }$. We wish to estimate the mean number of collisional events that the grains of mass $m$ must have had in their collisional history.

Each individual fragmenting collision of this cascade can be described by a distribution $g\left(m, m_{x}\right)$ of fragments $m$ from the largest one $m_{x}$ down to infinitely small ones. Let the normalization condition be given by

$\int_{0}^{m_{x}} g\left(m, m_{x}\right) \mathrm{d} m=1$.

Now we introduce a relation between $m_{x}$ and $m_{\max }$ given by $\mu=m_{x} / m_{\max }$. As each catastrophic collision in a cascade reduces the mass of the largest possible fragment by the factor $\mu$, the maximum number of collisions that a fragment of size $m$ might have undergone since the parent body $m_{\max }$ was disrupted is given by

$k_{\max }=\frac{\ln \left(m / m_{\max }\right)}{\ln \mu}$.

If we assume every collision to be described by the same $\mu$ and the same power-law dependence for

$$
g\left(m, m_{x}\right) \equiv(2-\eta) \frac{m_{x}^{\eta-2}}{m^{\eta-1}},
$$

but with a different absolute value of $m_{x}$, the resulting distribution after $k+1$ collisions is given by

$$
\begin{aligned}
& P_{\mathrm{k}}\left(m, m_{\max }\right)= \int_{m \mu^{-k}}^{m_{\max } \mu} g\left(m_{1}, m_{\max } \mu\right) \int_{m \mu^{1-k}}^{m_{1} \mu} g\left(m_{2}, m_{1} \mu\right) \int_{m \mu^{2-k}}^{m_{k-1} \mu} g\left(m_{3}, m_{2} \mu\right) \cdots \\
& \int_{m \mu^{-2}}^{m_{k}} g\left(m_{\mathrm{k}}, m_{\mathrm{k}-1} \mu\right) g\left(m, m_{\mathrm{k}} \mu\right) \\
& \mathrm{d} m_{\mathrm{k}} \cdots \mathrm{d} m_{3} \mathrm{~d} m_{2} \mathrm{~d} m_{1},
\end{aligned}
$$

where $P_{\mathrm{k}}$ provides the fraction of mass that falls in the range [m, $m+\mathrm{d} m]$ after $k+1$ collisions. If we change to logarithmic scaling of the integration variables, i.e. $\mathrm{d} m_{1}=m_{1} \mathrm{~d}\left(\ln \frac{m_{1}}{m}\right)$ and so on, and explicitly insert $g$, we obtain

$$
\begin{aligned}
P_{\mathrm{k}}\left(m, m_{\max }\right)= & (2-\eta)^{k+1} \int_{\ln \mu^{-k}}^{\ln \frac{\mu m_{\max }}{m}} \frac{\left(m_{\max } \mu\right)^{\eta-2}}{m_{1}^{\eta-1}} \int_{\ln \mu^{1-k}}^{\ln \frac{\mu m_{1}}{m}} \frac{\left(m_{1} \mu\right)^{\eta-2}}{m_{2}^{\eta-1}} \\
& \int_{\ln \mu^{2-k}}^{\ln \frac{\mu m_{2}}{m}} \frac{\left(m_{2} \mu\right)^{\eta-2}}{m_{3}^{\eta-1}} \cdots \int_{\ln \mu^{-2}}^{\frac{\mu m_{k-1}}{m}} \frac{\left(m_{k-1} \mu\right)^{\eta-2}}{m_{\mathrm{k}}^{\eta-1}} \frac{\left(m_{\mathrm{k}} \mu\right)^{\eta-2}}{m^{\eta-1}} \\
& \times m_{\mathrm{k}} \cdots m_{3} m_{2} m_{1} \\
& \mathrm{~d}\left(\ln \frac{m_{\mathrm{k}}}{m}\right) \cdots \mathrm{d}\left(\ln \frac{m_{3}}{m}\right) \mathrm{d}\left(\ln \frac{m_{2}}{m}\right) \mathrm{d}\left(\ln \frac{m_{1}}{m}\right) .
\end{aligned}
$$

Luckily, all integration variables disappear and the integrand is constant:

$P_{\mathrm{k}}\left(m, m_{\max }\right)=(2-\eta)^{k+1} \frac{1}{m}\left(\frac{\mu^{k+1} m_{\max }}{m}\right)^{\eta-2}$

$$
\begin{aligned}
& \times \int_{\ln \mu^{-k}}^{\ln \frac{\mu m_{\max }}{m}} \int_{\ln \mu^{1-k}}^{\ln } \int_{\ln \mu^{2-k}}^{\frac{\mu m_{1}}{m}} \cdots \int_{\ln \mu^{-2}}^{\ln \frac{\mu m_{2}}{m}} \ln \frac{\mu m_{k-1}}{m} \\
& \mathrm{~d}\left(\ln \frac{m_{\mathrm{k}}}{m}\right) \cdots \mathrm{d}\left(\ln \frac{m_{3}}{m}\right) \mathrm{d}\left(\ln \frac{m_{2}}{m}\right) \mathrm{d}\left(\ln \frac{m_{1}}{m}\right) .
\end{aligned}
$$

Now the variables are shifted starting with $m_{1} \rightarrow \mu^{k} m_{1}$ :

$$
\begin{aligned}
& P_{\mathrm{k}}\left(m, m_{\max }\right)=(2-\eta)^{k+1} \frac{1}{m}\left(\frac{\mu^{k+1} m_{\max }}{m}\right)^{\eta-2} \\
& \times \int_{0}^{\ln \frac{\mu^{k+1} m_{\max }}{m}} \int_{\ln \mu^{1-k}} \int_{\ln \mu^{2-k}}^{\frac{\mu^{1-k_{m_{1}}}}{m}} \cdots \int_{\ln \mu^{-2}}^{\ln } \\
& \mathrm{d}\left(\ln \frac{m_{\mathrm{k}}}{m}\right) \cdots \mathrm{d}\left(\ln \frac{m_{3}}{m}\right) \mathrm{d}\left(\ln \frac{m_{2}}{m}\right) \mathrm{d}\left(\ln \frac{m_{1}}{m}\right) .
\end{aligned}
$$

Substituting everything from $m_{1}$ to $m_{\mathrm{k}}$ in the same manner, we obtain

$$
\begin{aligned}
P_{\mathrm{k}}\left(m, m_{\max }\right)= & (2-\eta)^{k+1} \frac{1}{m}\left(\frac{\mu^{k+1} m_{\max }}{m}\right)^{\eta-2} \\
& \times \int_{0}^{\ln \frac{\mu^{k+1} m_{\max }}{m}} \int_{0}^{\ln } \int_{0}^{\frac{m_{1}}{m}} \cdots \int_{0}^{\ln \frac{m_{2}}{m}} \\
& \mathrm{~d}\left(\ln \frac{m_{\mathrm{k}}}{m}\right) \cdots \mathrm{d}\left(\ln \frac{m_{3}}{m}\right) \mathrm{d}\left(\ln \frac{m_{2}}{m}\right) \mathrm{d}\left(\ln \frac{m_{1}}{m}\right) .
\end{aligned}
$$

The integral equates the volume spanned by $0<x_{\mathrm{k}}<\cdots<x_{3}<$ $x_{2}<x_{1}<x_{0}=\ln \frac{\mu^{k+1} m_{\max }}{m}$, which is given by

$$
\int_{0}^{x_{0}=\ln } \int_{0}^{\frac{\mu^{k+1} m_{\max }}{m^{m}}} \int_{0}^{x_{1}} \cdots \int_{0}^{x_{\mathrm{k}}} \mathrm{d} x_{\mathrm{k}} \cdots \mathrm{d} x_{3} \mathrm{~d} x_{2} \mathrm{~d} x_{1}=\frac{\left[\ln \frac{\mu^{k+1} m_{\max }}{m}\right]^{k}}{k !} .
$$

The final result is

$P_{\mathrm{k}}\left(m, m_{\max }\right)=\frac{1}{m}(2-\eta)^{k+1}\left(\frac{\mu^{k+1} m_{\max }}{m}\right)^{\eta-2} \frac{\left[\ln \left(\frac{\mu^{k+1} m_{\max }}{m}\right)\right]^{k}}{k !}$

or

$P_{\mathrm{k}}\left(m, m_{\max }\right)=\frac{2-\eta}{m}\left(\frac{\mu^{k+1} m_{\max }}{m}\right)^{\eta-2} \frac{\left\{\ln \left[\left(\frac{\mu^{k+1} m_{\max }}{m}\right)^{2-\eta}\right]\right\}^{k}}{k !}$

For $k=0$, i.e. no intermediate collision, we are back at

$P_{0}\left(m, m_{\max }\right)=g\left(m, \mu m_{\max }\right)$.

To obtain the distribution of the total mass of fragments per mass decade, we have to evaluate

$\tilde{P}=m \cdot \ln (10) \cdot P$.

Figure A.1 shows examples for the distribution. The planetesimal radius $s_{\max }=10 \mathrm{~km}$ and the grain radius $s=100$ to $10 \mu \mathrm{m}$ correspond to the mass ratio $\log _{10}\left(m_{\max } / m\right) \sim 24$ to 27 , or $x$ from -3 to 0 . In this case, the distributions peak at $k=7$ to 10 . Therefore, we adopt $k=8$ for the bulk of the calculations. 
A. V. Krivov et al.: Clumps in debris disks, Online Material p 3
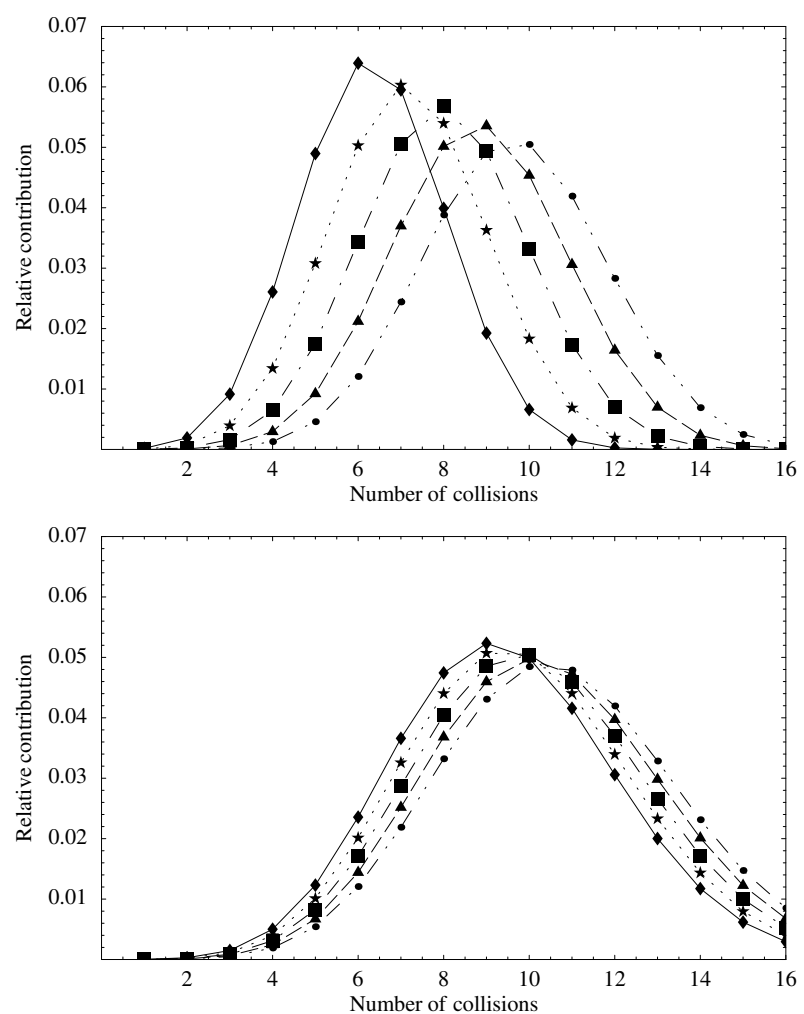

Fig. A.1. Probability distribution of particles with size $m$ originating from parent bodies of mass $m_{\max }=10^{27+x} \mathrm{~m}$ over the number of collisions they underwent. $x$ has values $-6,-3,0,3,6$ (peaks from left to right). $\eta=11 / 6$. The peak position is roughly proportional to $\log _{10}\left(m_{\max } / m\right) / 3.5$. (The lines just connect adjacent points for illustrative purpose, although the distribution is discrete in $k$. The distribution looks similar to a Poisson distribution but is different.) Top: $m_{x} / m_{\max }=\mu=0.1$, bottom: $\mu=0.5$. 